\title{
A Novel Verification Protocol to Restrict Unconstitutional Access of Information From Smart Card
}

\author{
Ajay Kumar Sahu, Raj Kumar Goel Institute of Technology and Management, India
}

Ashish Kumar, ITS Engineering College, India

\begin{abstract}
The services of the internet play an essential part in the daily life of the users. So, safety and confidentiality of the information are to be maintained to preserve user conviction in various services offered by network. The two-factor-based password verification techniques are used between remote server and legitimate users for verification over insecure channel. Several protocols have been suggested previously claiming their simplicity, privacy, safety, and robustness. The authors proved that their enhanced protocols are vulnerable to different attacks on the network and permit only authenticated users to update their password preserving traceability and identity. Analysis shows that no scheme has fulfilled all the security requirements and achieved entire goals. Therefore, in this article, a scheme has been presented to overcome these issues in the previous schemes to resist illegal access leading to misuse and achieve all the security requirements and goals. The safety analysis of the presented scheme has confirmed its performance in terms of reliability and safety.
\end{abstract}

\section{KEYWORDS}

Hash Function, Identity, Information Retrieval, Key Agreement, Mutual Authentication, Password, Security, Smart Card

\section{INTRODUCTION}

As time grows day by day, dependency of user in various technology increases which constituted a challenge regarding validity of the remote user. There are various types of attacks possible in the network which causes significant financial loss. Therefore, there is a requirement of some techniques to validate the legitimate users to an unsafe media such as Internet. The most commonly used technique is two factor based password verification. This protocol is susceptible to numerous attacks caused by human intellectual capacity of scheming and memorizing typical passwords.

Chip card based technique can be efficiently implemented in various password-based verification protocols (Lamport, 1991), (Gamal, 1985), (Kocher \& Jaffe, 1999), (Messerges, Dabbish, \& Sloan, 2002), (Chang C. C \& Wu T. C, 1993), (Hwang M. S \& Lee, 2000), (Kumar \& Gupta, 2011), (Xiong $\&$ Niu, 2014) and (Kumari \& Khan, 2013) easily. These have several applications like financial 
transactions, identity approval and accessing of remote services. To improve their feasibility, cards are confined in to limited size and cost. Various protocols has been reported (Tang, Hwang, \& Lee, 2002), (Chang \& Chang, 2005) and (Srivastva \& Sharma, 2012) in which user may update password without interacting with the server, however user's identity must be same in every login attempt. Moreover, the schemes based on variable identity (Das, Saxena, \& Gulati, 2004), (Wang, Liu, Xiao, \& Dan, 2009), (Chang and Chang, 2009), (Madhusudhan \& Mittal, 2012), (Chang, Tai \& Chang, 2013), (Khan et al., 2014), (Devgan \& Awasthi, 2016), (Chaudhary et al., 2015), (Wang et al., 2015), (Kharu et al., 2018), (Lu et al., 2016) and (Jung, Lee \& Kim, 2016) are less prone to attacks and promising.

Literature review of the various schemes shows that till date most of the presented schemes are unsafe to different attacks like pose attack, online password guessing attack, chip card misplaced attack, repetition attack and man in middle attack. Many of the above schemes need a lot of storage cost and computational cost which decreases the performance of the scheme. Various schemes are fail to achieve all the security parameters and goals; therefore a need arises to develop a protocol that fulfils the entire above criterion. Therefore, in this paper, the authors proposed a scheme as A Novel Verification Protocol to Restrict Unconstitutional Access of Information from Smart Card.

\section{NOTATIONS AND DESCRIPTION}

The following symbols/notations are preferred in this paper as described in Table 1.

\section{Scheme Design}

Initially, user enters his personal information to the terminal and sends towards the server for registration. Then user obtains chip card delivered by the server with security parameters. The

\section{Table 1. Symbols/Notations}

\begin{tabular}{|cl|}
\hline$S Y M B O L$ & DESCRIPTION \\
$\mathrm{u}_{\mathrm{i}}$ & User \\
$\mathrm{s}_{\mathrm{i}}$ & Server \\
$\mathrm{C} C_{\mathrm{i}}$ & Chip Card \\
$\mathrm{id}_{\mathrm{i}}$ & Identity of user \\
$\mathrm{c}_{\mathrm{i}} \mathrm{d}_{\mathrm{i}}$ & Dynamic identity \\
$\mathrm{Z}$ & Attacker \\
$\mathrm{p}_{\mathrm{w}} \mathrm{d}_{\mathrm{i}}$ & Password \\
$\mathrm{h}()$. & Hash Function \\
$\oplus$ & XOR Function \\
$\|$ & Concatenation Operation \\
$\alpha$ & User's Arbitrary Number \\
$\mathrm{mp}_{\mathrm{w}} \mathrm{d}_{\mathrm{i}}$ & Updated Password \\
$\beta_{\mathrm{i}}$ & Server's Arbitrary Number \\
$\mathrm{x}_{\mathrm{i}}, \mathrm{x}_{2}$ & Private key of Server, Secret number of Server \\
$\mathrm{t}_{1}$ & Current timestamp on Client Side \\
$\mathrm{t}_{2}$ & Current timestamp on Server Side \\
$\Delta \mathrm{t}$ & Maximum Communication Delay Time \\
$\gamma$ & Chip card's Random Number \\
$\mathrm{n}$ & Number of counts a user registers at the time of chip \\
& card lost
\end{tabular}


registration phase is required only once in this protocol unless user re-registers for unavoidable conditions. Login stage requires credentials given by a user and transmits this request towards server for accessing the resources. The transmission takes place only after both, server and user validates each other. The login and authentication process usually will be carried out several times. Password update stage and misplaced chip card re-registration stage provides the service to update its own password and after re-registration, resume these services offered by the server. The process flow diagram of system design is presented in figure 1.

\section{PROPOSED TWO FACTOR VERIFICATION SCHEME}

The proposed verification scheme consists of five different phases as: registration, login, verification, password update and revocation phase. It is simple, adequate and most secure variable identity based verification scheme to resist those attacks that exist in previous schemes. To reduce memorizing cost,

Figure 1. Process flow diagram of system design

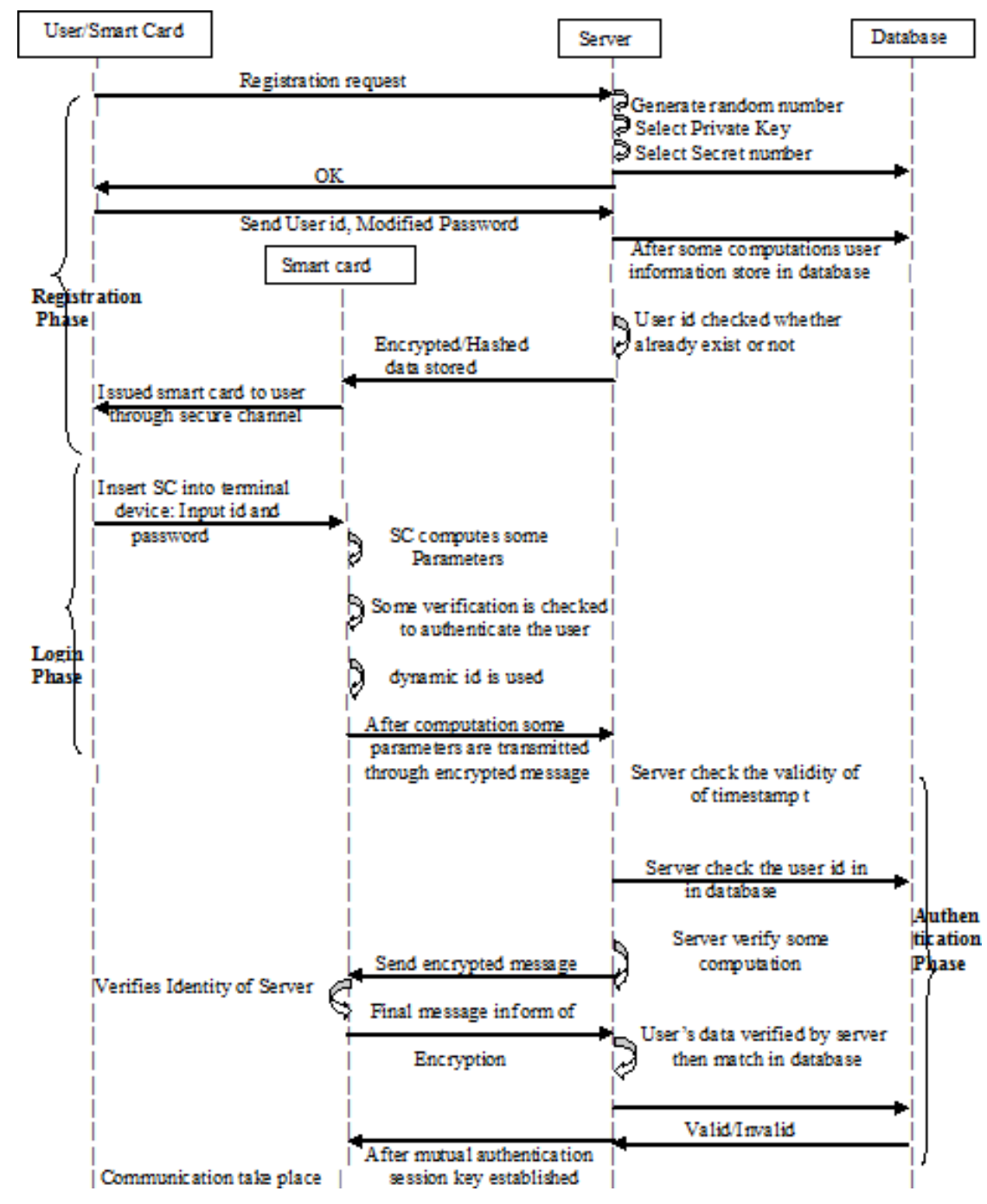


computational cost as well as to maintain its high performance and efficiency along with verified safety, scheme requires OR, Ex-OR, and elementary hash functions.

Within registration stage, chip card contains data which depends on the information produces by user and several credentials inserted through server. After the registration phase, both of user and server validate to each another then after successful mutual verification both server and user communicate to each other through session key agreement protocol. In the registration or authentication stage, initial validity is checked by smart card then transmits user's information towards server for more verification. In the presented scheme, user and server together validate to each other then after session key settled among both of them and transmission takes place. If the authentication of the server or user fails, this is recommended that the login stage is denied.

\section{Registration Stage}

In the beginning of this phase $\mathrm{u}_{\mathrm{i}}$ registers/re-registers with $\mathrm{s}_{\mathrm{i}}$ whenever wants some services from it. Assume $x_{1}$ and $x_{2}$ are private key and secret number of the server. Here $n$ specifies how many times a user registers by authentication server $\mathrm{s}_{\mathrm{i}}$. In some cases like chip card loss, theft or snatched, chip card can revoke through applying the value of $n$. This is saved in database of user's history on the server. The authentication server stores these secret key $\mathrm{x}_{1}$ and number $\mathrm{x}_{2}$ securely. The entire registration phase having a number of computation steps as follows:

Step 1: User $u_{i}$ select its own $i d_{i}$ along with $p_{w} d_{i}$ then choose an arbitrary no. $\alpha$. Compute $m p_{w} d_{i}=$ $h\left(\alpha \| p_{w} d_{i}\right)$ then transfers $\left\{i d_{i}, m p_{w} d_{i}\right\}$ towards $s_{i}$ through a protected medium.

Step 2: These registration credentials of user are verified by server and checks the database that selected identity is earlier present or not. If selected identity is match with another in database, $s_{i}$ warn $u_{i}$ to select another $i_{i}$. Server also investigates registration detail of $u_{i}$ then fixes the amount of $n$ accordingly. Specify value $n=0$ by $s_{i}$ for unique $u_{i}$, moreover specify $n=1$ by $s_{i}$ for re-registering user's into the server. In this way, each time of re-registration the value of $n$ is raised by 1 after that value of $\mathrm{n}$ and id ${ }_{i}$ will stored in the database.

Step 3: After getting $\left\{\mathrm{id}_{\mathrm{i}}, \mathrm{mp}_{\mathrm{w}} \mathrm{d}_{\mathrm{i}}\right\}$, server selects a random number $\beta_{\mathrm{i}}$, which is different for each user.

Step 4: Server estimates the values of $A_{i}=h\left(i d_{u}\left\|\beta_{i}\right\| m p_{w} d_{i}\right)$ where $i_{u}=\left(i d_{i} \| n\right)$.

Step 5: Then $s_{i}$ compute $B_{i}=h\left(h\left(i d_{i}\right) \| x_{1}\right) \oplus m p_{w} d_{i}, C_{i}=\beta_{i} \oplus h\left(h\left(i d_{i}\right) \| x_{1}\right) \oplus m p_{w} d_{i}$ and $D_{i}=\beta_{i} \oplus h\left(x_{2} \| x_{1}\right)$. After that stores $\left\{\mathrm{C}_{\mathrm{i}}, \mathrm{A}_{\mathrm{i}}, \mathrm{D}_{\mathrm{i}}, \mathrm{h}().\right\}$ into chip card then deliver chip card, $\left.\mathrm{B}_{\mathrm{i}}\right\}$ to $\mathrm{u}_{\mathrm{i}}$ through a protected channel.

Step 6: Subsequently obtaining \{chip card, $B_{i}$ \} from $s_{i}$, then $u_{i}$ computes $E_{i}=h\left(i d_{i} \| p w d_{i}\right) \oplus \alpha, F_{i}=$ $\mathrm{B}_{\mathrm{i}} \oplus \alpha$ and stores $\left\{\mathrm{E}_{\mathrm{i}}, \mathrm{F}_{\mathrm{i}}\right\}$ into chip card.

\section{Login Stage}

For obtaining services from $\mathrm{s}_{\mathrm{i}}$, user must login into the server by inserting its personal chip card into terminal then input its own $\mathrm{id}_{\mathrm{i}}$ as well as $\mathrm{pwd}_{\mathrm{i}}$. After that chip card compute successive steps as:

Step 1: Compute $\alpha=\mathrm{E}_{\mathrm{i}} \oplus \mathrm{h}\left(\mathrm{id}_{\mathrm{i}} \| \mathrm{p}_{\mathrm{w}} \mathrm{d}_{\mathrm{i}}\right), \mathrm{mp}_{\mathrm{w}} \mathrm{d}_{\mathrm{i}}=\mathrm{h}\left(\alpha \| \mathrm{p}_{\mathrm{w}} \mathrm{d}_{\mathrm{i}}\right), \mathrm{h}\left(\mathrm{h}\left(\mathrm{id}_{\mathrm{i}}\right) \| \mathrm{x}_{1}\right)=\mathrm{F}_{\mathrm{i}} \oplus \mathrm{m} \mathrm{p}_{\mathrm{w}} \mathrm{d}_{\mathrm{i}} \oplus \alpha, \beta_{\mathrm{i}}=\mathrm{C}_{\mathrm{i}} \oplus \mathrm{h}\left(\mathrm{h}\left(\mathrm{id}_{\mathrm{i}}\right)\right.$ $\left.\| \mathrm{x}_{1}\right) \oplus m \mathrm{p}_{\mathrm{w}} \mathrm{d}_{\mathrm{i}}$.

Step 2: The validity of the following equation is $A_{i}$ ? $=h\left(i d_{i}\left\|\beta_{i}\right\| m p_{w} d_{i}\right)$ is checked by chip card i.e it correct or not. If equation is not correct, chip card drops this session. If correct, chip card computes $\mathrm{h}\left(\mathrm{x}_{2} \| \mathrm{x}_{1}\right)=\beta_{\mathrm{i}} \oplus \mathrm{D}_{\mathrm{i}}, \mathrm{B}_{\mathrm{i}}=\mathrm{F}_{\mathrm{i}} \oplus \alpha$.

Step 3: Chip card acquires current time-stamp $t_{1}$ then evaluate $c_{i} d_{i}=h\left(i d_{i}\right) \oplus h\left(B_{i}\left\|\beta_{i}\right\| t_{1}\right), B_{i}=B_{i} \oplus h\left(\beta_{i} \| t_{1}\right)$, $\mathrm{G}_{\mathrm{i}}=\mathrm{B}_{\mathrm{i}} \oplus m \mathrm{p}_{\mathrm{w}} \mathrm{d}_{\mathrm{i}}$. Further take an arbitrary no. $\gamma$ then compute $\mathrm{H}_{\mathrm{i}}=\mathrm{h}\left(\mathrm{h}\left(\mathrm{id}_{\mathrm{i}}\right) \|_{\gamma}\right), \mathrm{I}_{\mathrm{i}}=\mathrm{G}_{\mathrm{i}} \oplus \mathrm{H}_{\mathrm{i}}, \mathrm{J}_{\mathrm{i}}=$ $\mathrm{h}\left(\mathrm{B}_{\mathrm{i}}\left\|\beta_{\mathrm{i}}\right\| \mathrm{H}_{\mathrm{i}} \| \mathrm{t}_{1}\right), \mathrm{K}_{\mathrm{i}}=\beta_{\mathrm{i}} \oplus\left(\mathrm{h}\left(\mathrm{x}_{2} \| \mathrm{x}_{1}\right) \| \mathrm{t}_{1}\right)$ then transmits $\left\{\mathrm{c}_{\mathrm{i}} \mathrm{d}_{\mathrm{i}}, \mathrm{B}_{\mathrm{i}}, \mathrm{J}_{\mathrm{i}}, \mathrm{K}_{\mathrm{i}}, \mathrm{t}_{1}, \mathrm{I}_{\mathrm{i}}\right\}$ to server via public channel. 


\section{Verification Stage}

After receiving login request message $\left\{\mathrm{c}_{\mathrm{i}} \mathrm{d}_{\mathrm{i}}, \mathrm{B}_{\mathrm{i}}, \mathrm{J}_{\mathrm{i}}, \mathrm{K}_{\mathrm{i}}, \mathrm{t}_{1}, \mathrm{I}_{\mathrm{i}}\right\}$ from $\mathrm{u}_{\mathrm{i}}$, server authenticates user and after proper mutual authentication, session key will be established as follows:

Step 1: Server acquires current time-stamp $t_{2}$ then justify whether $t_{1}$ is authentic means $t_{2}-t_{1} \leq \Delta t$. if $\mathrm{t}_{1}$ is not correct, server deny all the login request and drop this session.

Step 2: Analyze the database for obtaining the value of $n$ then determine $\mathrm{id}_{\mathrm{u}}=\left(\mathrm{id}_{\mathrm{i}} \| \mathrm{n}\right)$.

Step 3: If timestamp $t_{1}$ is valid, server continues to calculate $\beta_{i}=K_{i} \oplus\left(h\left(x_{2} \| x_{1}\right) \| t_{1}\right), B_{i}=B_{i} \oplus h\left(\beta_{i} \| t_{1}\right)$, $\mathrm{h}\left(\mathrm{id}_{\mathrm{i}}\right)=\mathrm{c}_{\mathrm{i}} \mathrm{d}_{\mathrm{i}} \oplus \mathrm{h}\left(\mathrm{B}_{\mathrm{i}}\left\|\beta_{\mathrm{i}}\right\| \mathrm{t}_{1}\right), \mathrm{G}_{\mathrm{i}}^{*}=\mathrm{h}\left(\mathrm{h}\left(\mathrm{id}_{\mathrm{i}}\right) \| \mathrm{x}_{1}\right), \mathrm{H}_{\mathrm{i}}{ }^{*}=\mathrm{G}_{\mathrm{i}}{ }^{*} \oplus \mathrm{I}_{\mathrm{i}}$. Then verify the equation $\mathrm{J}_{\mathrm{i}}$ ? = $\mathrm{h}\left(\mathrm{B}\left\|\beta_{i}\right\| \mathrm{H}_{i} * \|_{1}\right)$ holds or not.

Step 4: If above equation is verified, server acquires current time-stamp $t_{3}$ then determine $a=$ $h\left(G_{i} *\left\|\beta_{i}\right\| t_{3}\right)$, further server transmits $\left\{a, t_{3}\right\}$ towards user.

Step 5: After receiving $\left\{a, t_{3}\right\}$ from server, chip card confirm the accuracy of $t_{3}$. If timestamp $t_{3}$ is correct, it will check the equation $\mathrm{a} ?=\mathrm{h}\left(\mathrm{G}_{\mathrm{i}}\left\|\beta_{\mathrm{i}}\right\| \mathrm{t}_{3}\right\}$ holds or not. If this equation is correct, both $\mathrm{u}_{\mathrm{i}}$ and $s_{i}$ mutual validate to one another otherwise, this phase will be aborted by the server.

Step 6: Finally, server and user agreed upon a similar session key. The computed session key for the user is $\mathrm{s}_{\mathrm{k}}=\mathrm{h}\left(\mathrm{G}_{\mathrm{i}}\left\|\beta_{\mathrm{i}}\right\| \mathrm{t}_{1}\left\|\mathrm{t} \mathrm{t}_{3}\right\| \mathrm{h}\left(\mathrm{x}_{2} \| \mathrm{x}_{1}\right) \| \mathrm{H}_{\mathrm{i}}\right)$ and server $\mathrm{s}_{\mathrm{k}}^{*}=\mathrm{h}\left(\mathrm{G}_{\mathrm{i}}{ }^{*}\left\|_{\mathrm{i}_{1}}\right\| \mathrm{t}_{1}\left\|\mathrm{t}_{3}\right\| \mathrm{h}\left(\mathrm{x}_{2} \| \mathrm{x}_{1}\right) \| \mathrm{H}_{\mathrm{i}}^{*}\right)$ respectively.

This session key is used by user for secret transmission through encrypting and decrypting these messages via a secure channel.

\section{Password Update Stage}

Considering safety parameters if any user desire to modify its own password $p_{w} d_{i}$ with new password $p_{w} d_{n e w}$ in the system, user insert its own chip card into terminal of card reader further input its own $\mathrm{id}_{\mathrm{i}}$ and $\mathrm{p}_{\mathrm{w}} \mathrm{d}_{\mathrm{i}}$. The following computation has been performed by the chip card without involvement of remote server $\mathrm{S}$.

Step 1: Chip card computes the value of $\alpha=E_{i} \oplus h\left(i d_{i} \| p_{w} d_{i}\right), m_{w} d_{i}=h\left(\alpha \| p_{w} d_{i}\right), h\left(h\left(i d_{i}\right) \| x_{1}\right)=$ $\mathrm{F}_{\mathrm{i}} \oplus m \mathrm{p}_{\mathrm{w}} \mathrm{d}_{\mathrm{i}} \oplus \alpha, \beta_{\mathrm{i}}=\mathrm{C}_{\mathrm{i}} \oplus \mathrm{h}\left(\mathrm{id} \mathrm{d}_{\mathrm{i}} \| \mathrm{x}_{1}\right) \oplus \mathrm{mp} \mathrm{p}_{\mathrm{w}} \mathrm{d}_{\mathrm{i}}$ then test this equation $\mathrm{A}_{\mathrm{i}}$ ? $=\mathrm{h}\left(\mathrm{id}_{\mathrm{i}}\left\|\beta_{\mathrm{i}}\right\| \mathrm{mp}_{\mathrm{w}} \mathrm{d}_{\mathrm{i}}\right)$ is correct or not.

Step 2: In case that the condition is true, then remote user is granted to modify his $\mathrm{pwd}_{\mathrm{i}}$ otherwise session is aborted.

Step 3: Then chip card computes $m p_{w} d_{i}{ }^{\text {new }}=h\left(\alpha \| p_{w} d_{i}{ }^{\text {new }}\right), E_{i}{ }^{\text {new }}=h\left(i d_{i} \| p_{w} d_{i}{ }^{\text {new }}\right) \oplus \alpha, F_{i}{ }^{\text {new }}=$ $\mathrm{F}_{i} \oplus m p_{w} d_{i} \oplus m p_{w} d_{i}^{\text {new }}, C_{i}^{\text {new }}=C_{i} \oplus m p_{w} d_{i} \oplus m p_{w} d_{i}^{\text {new }}, A_{i}^{\text {new }}=h\left(i d_{u}\left\|\beta_{i}\right\| m p_{w} d_{i}^{\text {new }}\right)$ and replaces the old $\left\{E_{i}, A_{i}, F_{i}, C_{i}\right\}$ with $\left\{E_{i}^{\text {new }}, A_{i}^{\text {new }}, F_{i}^{\text {new }}, C_{i}^{\text {new }}\right\}$ respectively. Now, the modified password has changed successfully and this phase is terminated.

\section{Revocation Stage}

This stage is initialised in the case of chip card lost, damage or misplaced situation. For revocation of chip card user forwards a request towards server. Moreover, after receiving the request server ask several credentials from user to check the authenticity, like Adhaar number, Mobile OTP, Birth date, Mother's maiden name or any other user's known value. Furthermore, after checking the validity of this revocation request created by end user, server modifies current value of $n$ for re-registering the chip card. In each time of misplaced or lost chip card case, $\mathrm{n}$ is incremented by 1 . Afterwards, user re-register's himself to server without updating its own identity. For the revocation of this card, it is desired from user that don't avail any earlier values like earlier password, arbitrary number otherwise by availing same values which was already stored within misplaced or stolen smart card anybody may pose as a server's legitimate user. 


\section{IMPLEMENTATION AND CODING}

The presented scheme has been coded in PYTHON language. The screen shots of the following phases are described as shown in Figures 2-5.

The figure 2 represents all the five phases in single screen. Here phase 1 represents registration phase, phase 2 represents login phase, phase 3 represents verification phase, phase 4 represents password change phase and in the last phase 5 represents revocation phase of lost smart card.

\section{FORMAL SAFETY VALIDATION WITH AVISPA TOOL}

AVISPA is described as a pushdown software tool used to validate the internet security protocols. It supports High Level Protocol Specification Languages (HLPSL) and offers the formal safety

Figure 2. Different Phases of Proposed Protocol

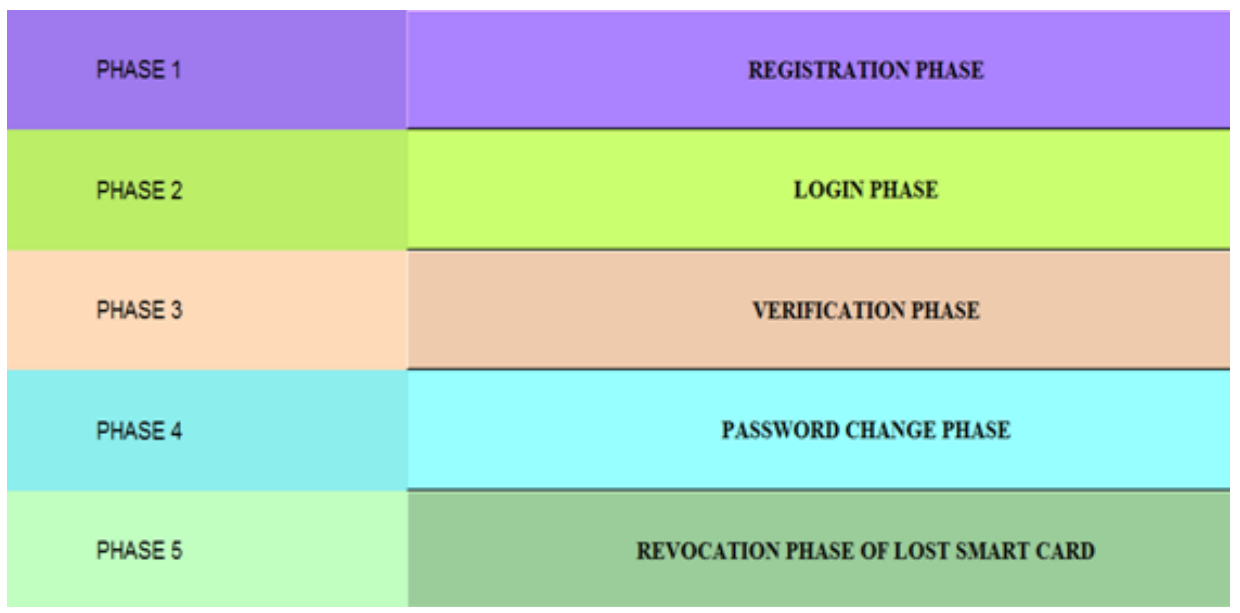

Figure 3. Registration Phase of Proposed Protocol

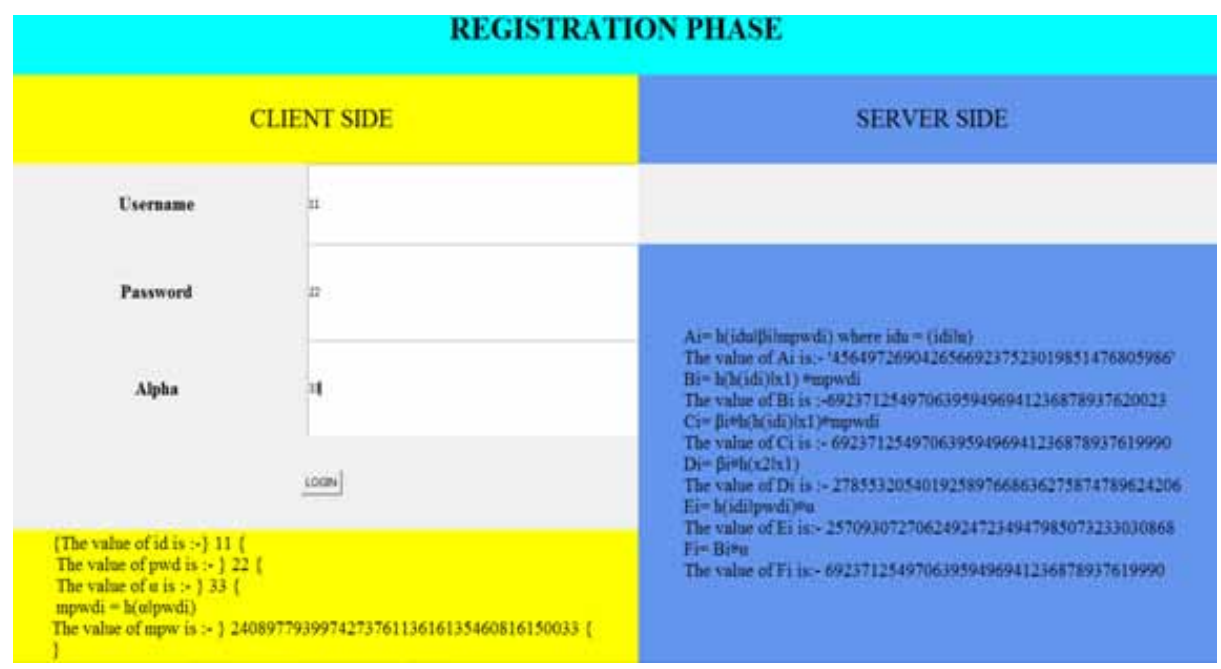


Figure 4. Login Phase of Proposed Protocol

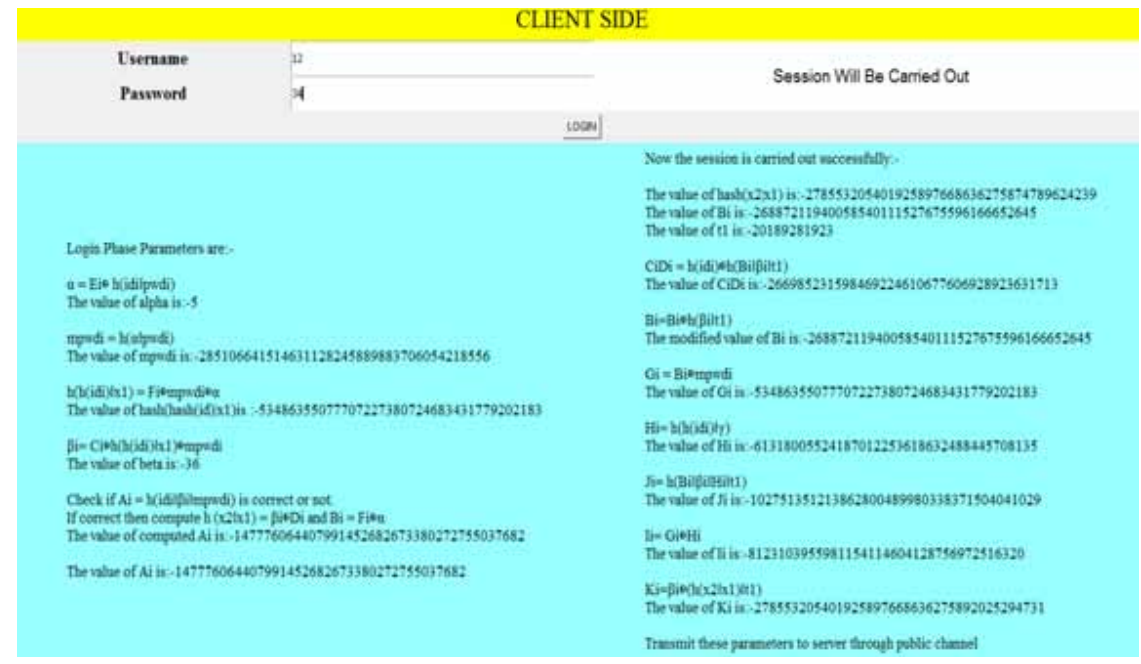

Figure 5. Password Change Phase of Proposed Protocol

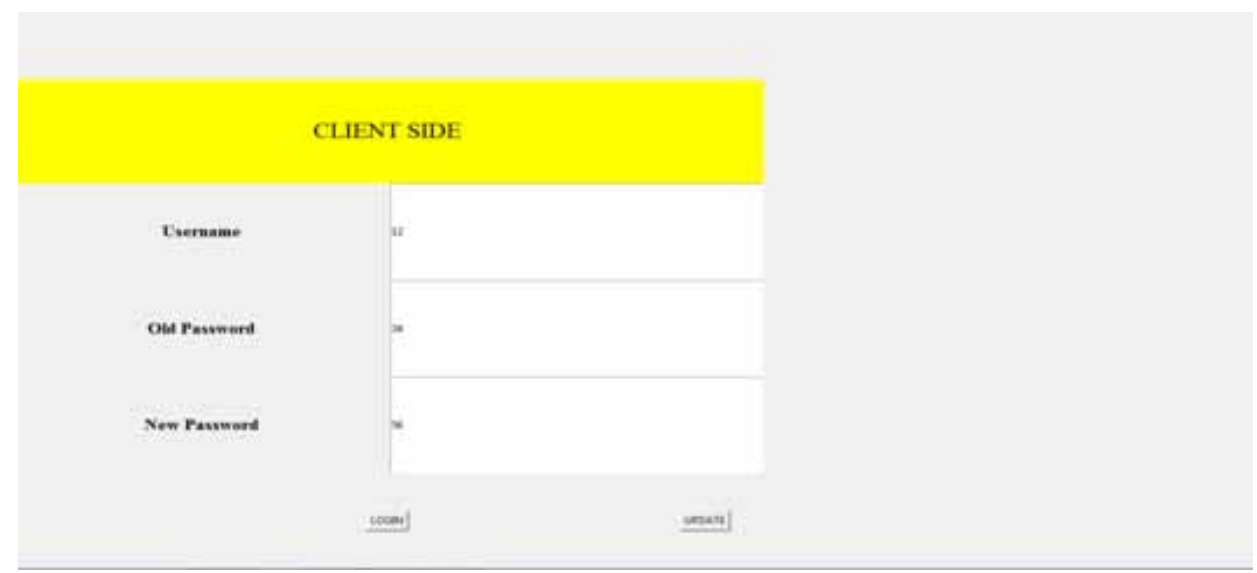

Figure 6. Components of AVISPA tool

High-Level Protocol Specification Language (HLPSL)

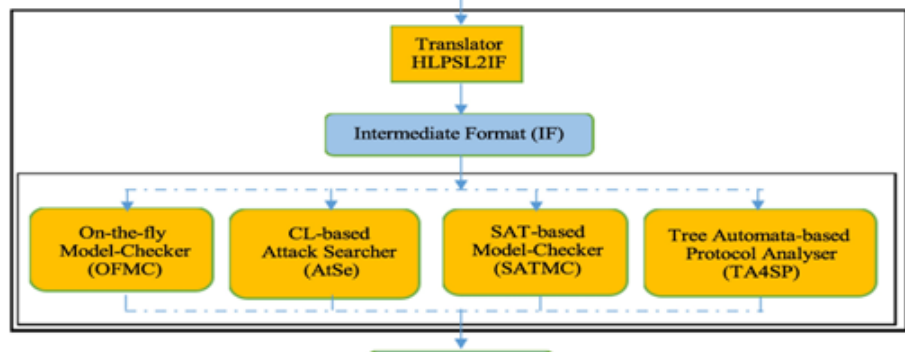

Output Format

(OF) 
verification with simulated protocol. Using AVISPA, their simulation results shows that whether presented protocol is secure against the different attacks.

In order to verify the safety of presented protocol, AVISPA software tool is apply to simulate results. Therefore, presented protocol translates into HLPSL code. In this paper, the role of user, server, the session, goal and environment coded in HLPSL specification is presented below. After execution this HLPSL code into AVISPA tool, simulation results confirm that presented protocol is SAFE with respect to different active as well as passive attacks.

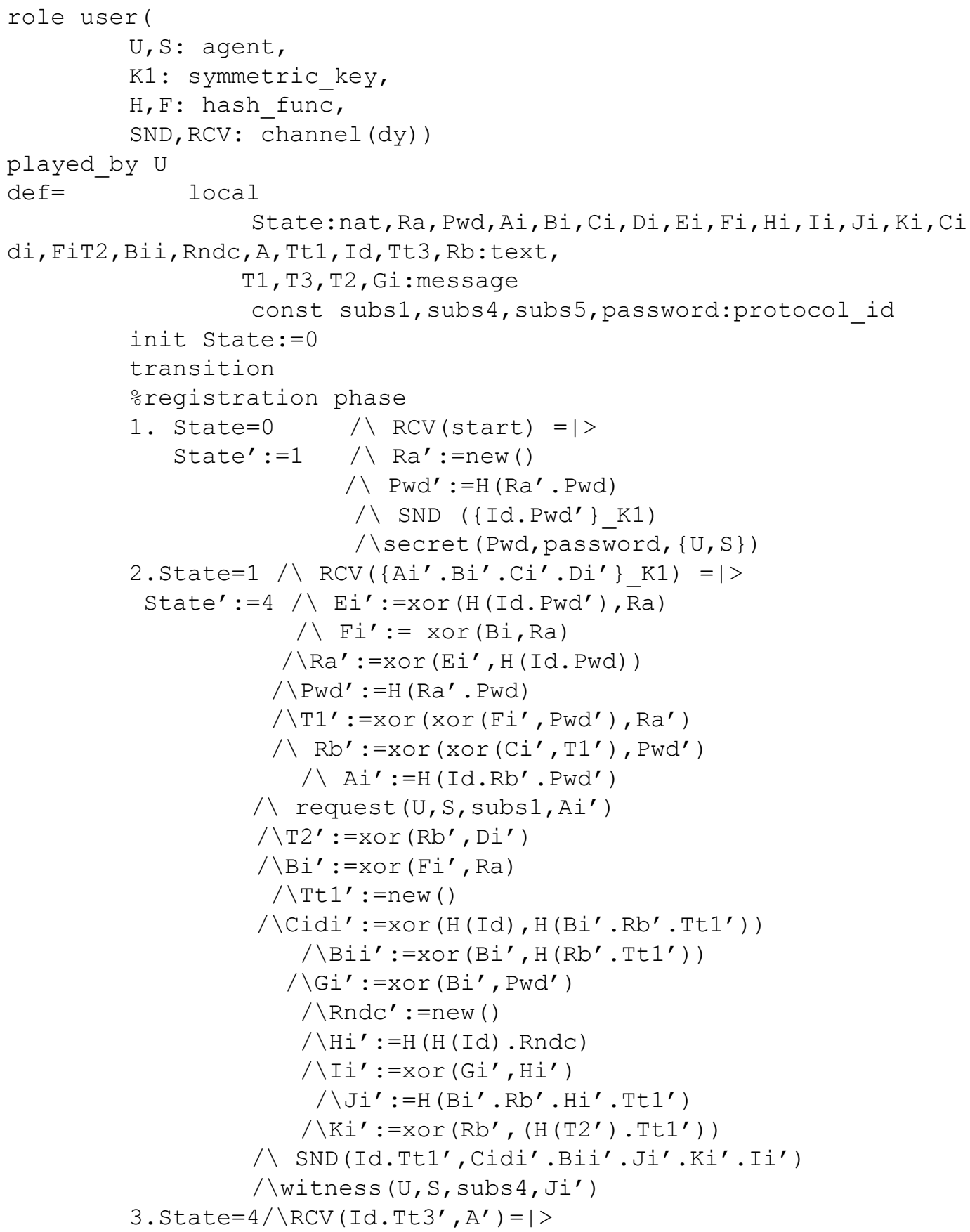




$$
\begin{aligned}
\text { State }^{\prime}:=6 & / \mathrm{A}^{\prime}:=\mathrm{H}\left(\mathrm{Gi} \cdot \mathrm{Rb} . \mathrm{Tt} 3^{\prime}\right) \\
& / \backslash \text { request }\left(\mathrm{U}, \mathrm{S}, \operatorname{subs} 5, \mathrm{~A}^{\prime}\right)
\end{aligned}
$$

end role

role server (

$$
\mathrm{U}, \mathrm{S} \text { : agent, }
$$

K1: symmetric_key,

$\mathrm{H}, \mathrm{F}$ : hash_func,

SND, RCV: channel (dy))

played_by $S$

def $=$

\section{local}

State: nat, Ra, Pwd, Ai, Bi, Ci, Di, Ei, Hi, Ii, Ji, Ki, Cidi, Fi, Rb, Rndc, Hid, A, X1, X2, Tt1, Bii, Tt3, Id: text,

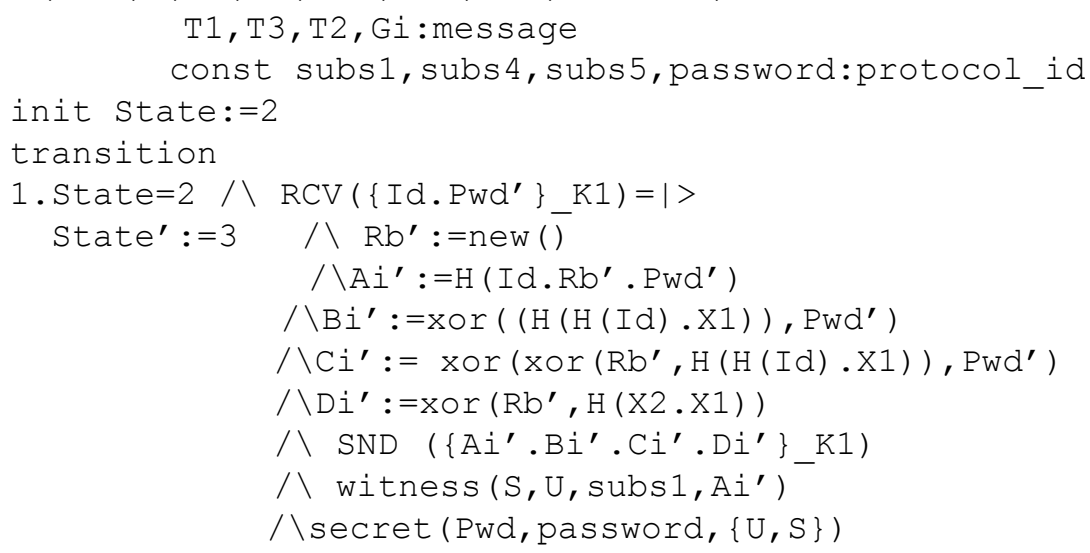

$\mathrm{T} 1, \mathrm{~T} 3, \mathrm{~T} 2, \mathrm{Gi}$ :message

const subs1, subs4, subs5, password:protocol_id

init state: $=2$

end role

role session (

$\mathrm{U}, \mathrm{S}$ : agent,

$\mathrm{K}$ : symmetric_key,

MD1, MD2: hash_func)

def $=$

local SENDU, SENDS, RECS, RECU: channel (dy) composition user (U, S, K, MD1, MD2, SENDU, RECS) / server (U, S, K, MD1, MD2, SE NDS, RECU)

end role 


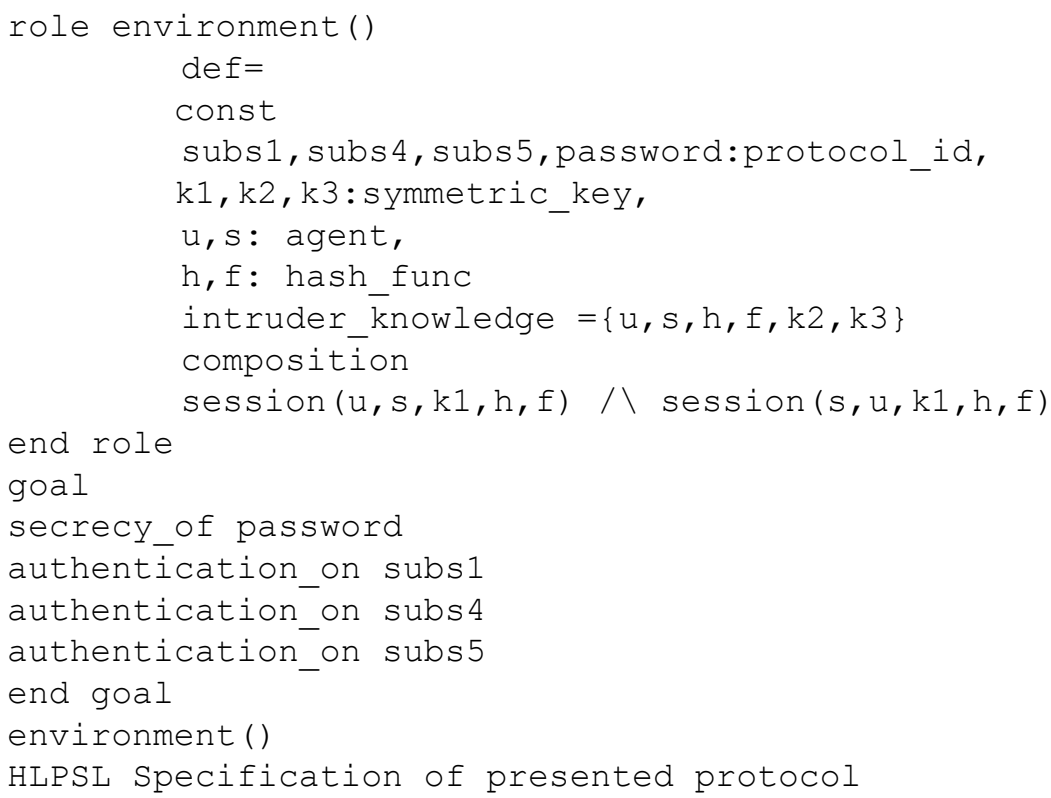

\section{PERFORMANCE EVALUATION}

The following section determines and estimates different performance criterion of presented protocol in terms of memory space, transmission cost and computation cost with other protocols suggested by various researchers. In this paper, time complexity of hash operation is represented as $t_{h}$ and XOR operation as $t_{\oplus}$. Here, the authors suppose that some parameters as arbitrary numbers, secret numbers, password, time-stamps and identity are 128 bits. The performance evaluation regarding different schemes is shown in table 2.

Table 2. Efficiency Comparison related with Memory Space in Smart Card requirement (in bits), transmission cost (in bits) and Computational complexity cost (in bits)

\begin{tabular}{|l|l|l|l|l|l|}
\hline \multicolumn{1}{|c|}{ Protocols } & \multicolumn{1}{|c|}{$\begin{array}{c}\text { Presented } \\
\text { Protocol }\end{array}$} & \multicolumn{1}{|c|}{$\begin{array}{c}\text { Kumari et al., } \\
(\mathbf{2 0 1 4})\end{array}$} & \multicolumn{1}{c|}{$\begin{array}{c}\text { Qiu S. et al., } \\
(\mathbf{2 0 1 8})\end{array}$} & \multicolumn{1}{c|}{$\begin{array}{c}\text { Mishra D. } \\
\text { (2015) }\end{array}$} & \multicolumn{1}{c|}{$\begin{array}{c}\text { Jung J. et } \\
\text { al., (2018) }\end{array}$} \\
\hline $\begin{array}{l}\text { Memory Space in smart } \\
\text { card (bits) }\end{array}$ & $6 * 128=768$ bits & $6 * 128=768$ bits & $\begin{array}{l}7 * 128=896 \\
\text { bits }\end{array}$ & $\begin{array}{l}4 * 128=512 \\
\text { bits }\end{array}$ & $\begin{array}{l}4 * 128=512 \\
\text { bits }\end{array}$ \\
\hline Transmission Cost in (bits) & $\begin{array}{l}8 * 128=1024 \\
\text { bits }\end{array}$ & $7 * 128=896$ bits & $\begin{array}{l}3 * 128=384 \\
\text { bits }\end{array}$ & $\begin{array}{l}4 * 128=512 \\
\text { bits }\end{array}$ & $\begin{array}{l}4 * 128=512 \\
\text { bits }\end{array}$ \\
\hline Computational Complexity Cost & $2 \mathrm{t}_{\mathrm{h}}+2 \mathrm{t}_{\oplus}$ & $1 \mathrm{t}_{\mathrm{h}}+2 \mathrm{t}_{\oplus}$ & 0 & $1 \mathrm{t}_{\mathrm{h}}$ & $1 \mathrm{t}_{\mathrm{h}}$ \\
\hline $\begin{array}{l}\text { Registration Phase (User } \\
\text { Side) }\end{array}$ & $6 \mathrm{t}_{\mathrm{h}}+4 \mathrm{t}_{\oplus}$ & $4 \mathrm{t}_{\mathrm{h}}+3 \mathrm{t}_{\oplus}$ & $4 \mathrm{t}_{\mathrm{h}}+2 \mathrm{t}_{\oplus}$ & $2 \mathrm{t}_{\mathrm{h}}+2 \mathrm{t}_{\oplus}$ & $5 \mathrm{t}_{\mathrm{h}}+1 \mathrm{t}_{\oplus}$ \\
\hline $\begin{array}{l}\text { Registration Phase (Server } \\
\text { Side) }\end{array}$ & $12 \mathrm{t}_{\mathrm{h}}+11 \mathrm{t}_{\oplus}$ & $8 \mathrm{t}_{\mathrm{h}}+10 \mathrm{t}_{\oplus}$ & $4 \mathrm{t}_{\mathrm{h}}+3 \mathrm{t}_{\oplus}$ & $2 \mathrm{t}_{\mathrm{h}}+2 \mathrm{t}_{\oplus}$ & $5 \mathrm{t}_{\mathrm{h}}+3 \mathrm{t}_{\oplus}$ \\
\hline Login Phase & $8 \mathrm{t}_{\mathrm{h}}+4 \mathrm{t}_{\oplus}$ & $6 \mathrm{t}_{\mathrm{h}}+3 \mathrm{t}_{\oplus}$ & $6 \mathrm{t}_{\mathrm{h}}+1 \mathrm{t}_{\oplus}$ & $6 \mathrm{t}_{\mathrm{h}}+2 \mathrm{t}_{\oplus}$ & $6 \mathrm{t}_{\mathrm{h}}+9 \mathrm{t}_{\oplus}$ \\
\hline Authentication Phase & $9 \mathrm{t}_{\mathrm{h}}+10 \mathrm{t}_{\oplus}$ & $6 \mathrm{t}_{\mathrm{h}}+7 \mathrm{t}_{\oplus}$ & $4 \mathrm{t}_{\mathrm{h}}+3 \mathrm{t}_{\oplus}$ & $2 \mathrm{t}_{\mathrm{h}}+2 \mathrm{t}_{\oplus}$ & $7 \mathrm{t}_{\mathrm{h}}+2 \mathrm{t}_{\oplus}$ \\
\hline Password Change Phase & $37 \mathrm{t}_{\mathrm{h}}+31 \mathrm{t}_{\oplus}$ & $25 \mathrm{t}_{\mathrm{h}}+25 \mathrm{t}_{\oplus}$ & $18 \mathrm{t}_{\mathrm{h}}+9 \mathrm{t}_{\oplus}$ & $24 \mathrm{t}_{\mathrm{h}}+25 \mathrm{t}_{\oplus}$ & $24 \mathrm{t}_{\mathrm{h}}+15 \mathrm{t}_{\oplus}$ \\
\hline $\begin{array}{l}\text { Sum of Computational } \\
\text { Cost }\end{array}$
\end{tabular}




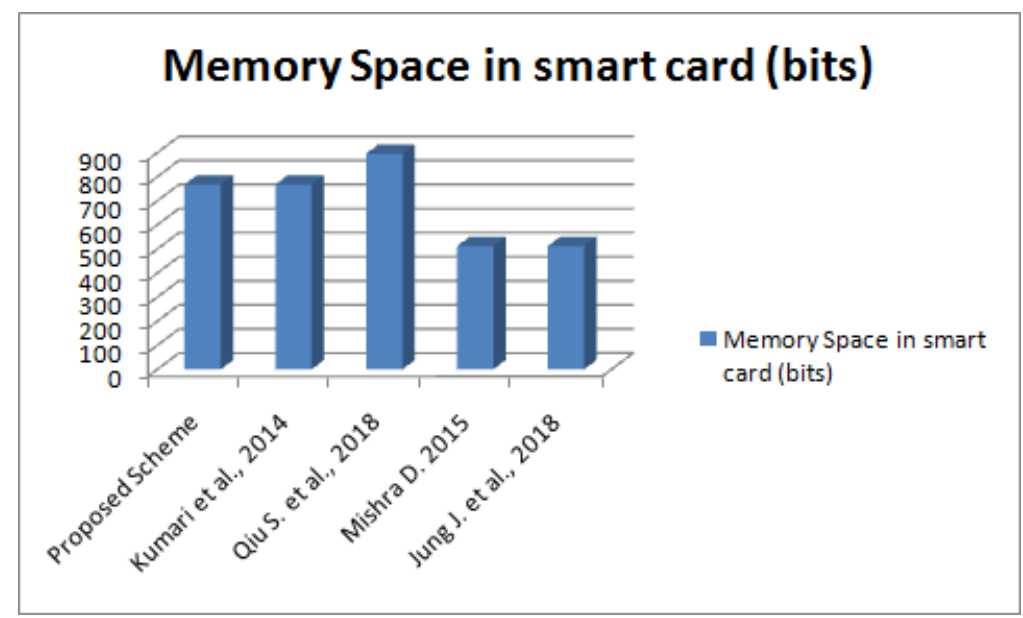

The memory space is described as total number of parameters stored in chip card. In this paper, total 6 variables like $\left\{\mathrm{A}_{\mathrm{i}}, \mathrm{C}_{\mathrm{i}}, \mathrm{D}_{\mathrm{i}}, \mathrm{E}_{\mathrm{i}}, \mathrm{F}_{\mathrm{i}}\right.$, hash $\}$ are stored in chip card's memory. Therefore, memory space required by chip card to store these parameters is $6^{*} 128=768$ bits.

Figure 8 shows the correlation graph of memory space needed in smart card (in bits) of presented scheme along with different other similar schemes.

In this scheme, to evaluate transmission cost, total 6 parameters $\left\{\mathrm{c}_{\mathrm{i}} \mathrm{d}_{\mathrm{i}}, \beta_{\mathrm{i}}, \mathrm{J}_{\mathrm{i}}, \mathrm{K}_{\mathrm{i}}, \mathrm{t}_{1}, \mathrm{I}_{\mathrm{i}}\right\}$ are required in login request, hence total bits computed as $6^{*} 128=768$ bits. Furthermore, in mutual verification total number of parameters used are $\left\{\mathrm{a}, \mathrm{t}_{3}\right\}$, requires $2 * 128=256$ bits. Hence, overhead for transmission becomes $=6 * 128+2 * 128=1024$ bits. Figure 9 presents the comparison graph of transmission cost in bits of presented scheme along with various other similar schemes.

In this scheme, registration phase requires two hash functions along with two XOR function in user side. Therefore, $2 \mathrm{t}_{\mathrm{h}(\mathrm{)})}+2 \mathrm{t}_{\oplus}$ is the computational complexity in user's side. Correspondingly, server requires six hash functions along with four XOR function during the registration phase, therefore, at server side, computational complexity is $6 \mathrm{t}_{\mathrm{h}(.)}+4 \mathrm{t}_{\oplus}$.

Figure 8. Transmission Cost (in bits) Comparison Graph

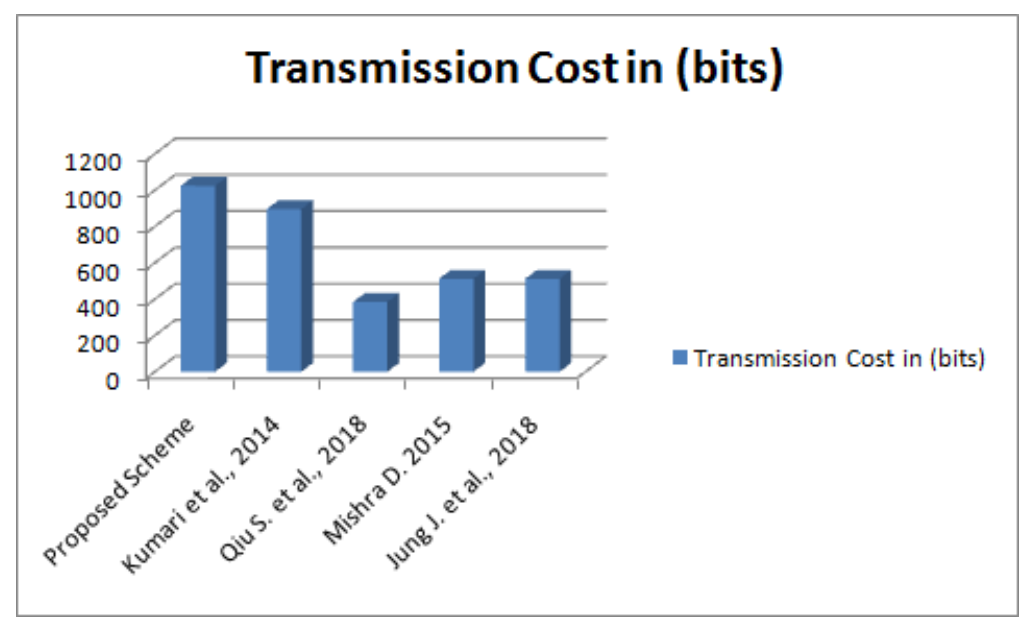




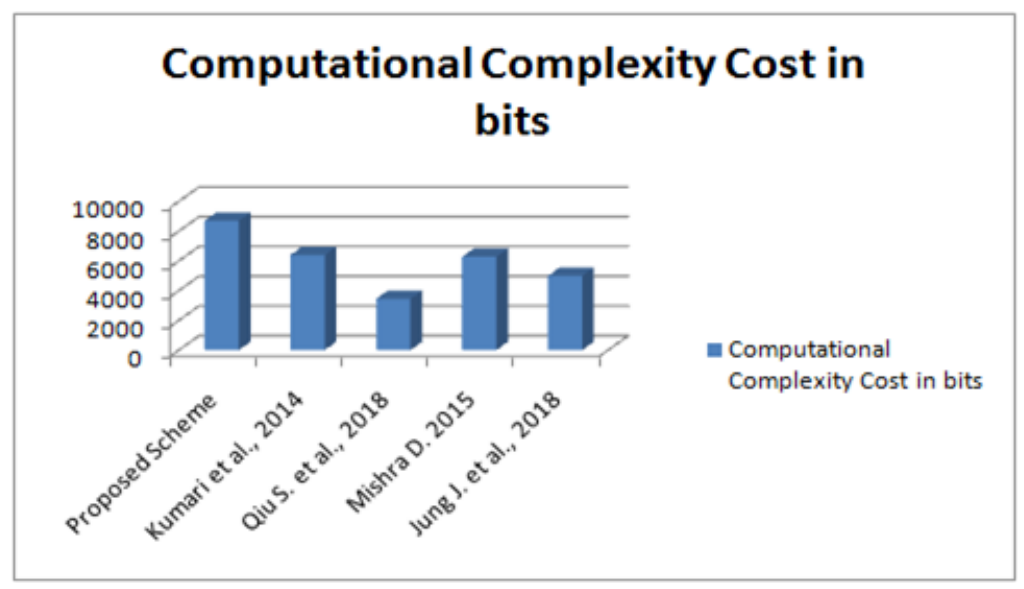

In this scheme, login stage requires 12 hash functions along with 11 XOR functions for login request. Hence, $12 \mathrm{t}_{\mathrm{h}(.)}+11 \mathrm{t}_{\oplus}$ is the computational cost for login message.

In this scheme, mutual authentication phase requires 1 hash function and server requires 7 hash functions along with 4 XOR functions. Therefore, total computational cost for authentication phase requires $8 \mathrm{~h}_{(.)}+4 \mathrm{t}_{\oplus}$ operations.

Figure 9 represents comparison graph of computational complexity evaluation cost in (bits) of this scheme corresponding with various other similar schemes.

\section{CONCLUSION}

In this paper, the presented scheme ensures security, privacy and confidentiality of a user. This scheme is an improvement over all the schemes presented in the literature review. After analysis, it is observed that earlier work is unsafe for practical applications because all security parameters can be easily obtained by the challenger and vulnerable to smart card misplaces violation as well as user un-traceability violation attack. Moreover, an adversary can get server's secret key, password of the entire registered user's and also the session key of the server, may also be obtained by an adversary which may lead to destroying the whole system. The presented scheme has been coded in PYTHON language and tested into AVISPA tool. The simulation results concluded that presented protocol is safe against entire active and passive attacks and achieve all the goals. The efficiency comparison of the scheme has confirmed its feasibility and performance to the practical approach. The presented scheme can be applied in such applications which providing privacy protection with low-computationability devices. Thus, our idea is practically more acceptable to operate secure remote access over the public environment as well as may be simply integrated into various types of services such as Military, Academics, Aeronautics, Banking, Crime control departments and Business applications. 


\section{REFERENCES}

Chang, C. C., \& Wu, T. C. (1993). Remote Password Authentication with Smartcards. Proceeding of Computers and Digital Techniques, 138(3), 165-168. doi:10.1049/ip-e.1991.0022

Chang, Y. F., \& Chang, C. C. (2005). Authentication Schemes with No Verification Table. Applied Mathematics and Computation, 167(2), 820-832. doi:10.1016/j.amc.2004.06.118

Chang, Y. F., \& Chang, H. C. (2009). Security of Dynamic ID-based Remote User Authentication Scheme. Fifth International Joint Conference on INC, IMS and IDC, 2108-2110.

Chang, Y. F., Tai, W. L., \& Chang, H. C. (2013). Untraceable Dynamic-Identity-based Remote User Authentication Scheme with Verifiable Password Update. International Journal of Communication Systems, 27(11), 3430-3440. doi:10.1002/dac. 2552

Chaudhary, S. A., Farash, M. S., N., Kumari, S., \& Khan, M. K. (2015). An Enhanced Privacy Preserving Remote User Authentication Scheme with Provable Security. The Journal of Security Communication Networks.

Das, M. L., Saxena, A., \& Gulati, V. P. (2004). A Dynamic ID-based Remote User Authentication Scheme. IEEE Transactions on Consumer Electronics, 50(2), 639-931. doi:10.1109/TCE.2004.1309441

Devgan, S., \& Awasthi, A. K. (2016). Security Enhancement of an Improved Remote User Authentication Scheme with Key Agreement. Wireless Personal Communications.

El Gamal, T. (1985). A Public-key Cryptosystem and a Signature Scheme based on Discrete Algorithms. IEEE Transactions on Information Theory, 31(4), 469-472. doi:10.1109/TIT.1985.1057074

Hwang, M. S., \& Li, L. H. (2000). New Remote User Authentication Scheme using Smart Cards. IEEE Transactions on Consumer Electronics, 46(1), 28-30. doi:10.1109/30.826377

Jung, J., Lee, D., \& Kim, J. (2016), Cryptanalysis and Improvement of Efficient Password-based User Authentication Scheme using Hash Function. ACM. doi:.<ALIGNMENT.qj></ALIGNMENT>10.1145/2857546.2857570

Jung, J., Lee, D., Lee, H., \& Won, D. (2018). Security Enhanced Anonymous User Authenticated Key Agreement Scheme using Smart Card. Journal of Electronic Science and Technology, 16(1), 45-49.

Khan, M. K., Kumari, S., Wang, X. M., \& Kumar, R. (2014). Dynamic ID-based Authentication Scheme. Proceeding of 12th International Conference on Dependable, Autonomic and Secure Computing (DASC), 347-361.

Khari, M., Shrivastava, G., Gupta, S., \& Gupta, R. (2018). Role of Cyber Security in Today's Scenario. In Cyber Security and Threats: Concepts, Methodologies, Tools, and Applications (pp. 1-15). IGI Global. doi:10.4018/9781-5225-5634-3.ch001

Kocher, P., Jaffe, J., \& Jun, B. (1999). Differential Power Analysis. Advances in Cryptology-CRYPTO, 388-397.

Kumar, M., Gupta, M. K., \& Kumari, S. (2011). An Improved Efficient Remote Password Authentication Scheme with Smart Card over Insecure Networks. International Journal of Network Security, 13(3), 167-177.

Kumari, S., \& Khan, M. K. (2013). Cryptanalysis and Improvement of a Robust Smart-Card based Remote User Password Authentication Scheme. International Journal of Communication Systems, 3939-3955.

Kumari, S., Khan, M. K., \& Li, X. (2014). An Improved Remote User Authentication Scheme with Key Agreement. Computers \& Electrical Engineering, 40(6), 1997-2012. doi:10.1016/j.compeleceng.2014.05.007

Lamport, L. (1991). Password Authentication with Insecure Communication. Communications of the ACM, 24(11), 770-772. doi:10.1145/358790.358797

Lu, Y., Li, L., Peng, H., \& Yang, Y. (2016). A Secure and Efficient Mutual Authentication Scheme for Session Initiation Protocol, Peer-to-Peer Net. App., 9(2), 449-459.

Madhusudhan, R., \& Mittal, R. C. (2012). Dynamic ID-based Remote User Password Authentication Schemes using Smart Cards. A Review. Journal of Network and Computer Applications, 35(4), 1235-1248. doi:10.1016/j. jnca.2012.01.007 
Messerges, T. S., Dabbish, E. A., \& Sloan, R. H. (2002). Examining Smart-card Security under the Threat of Power Analysis Attacks. IEEE Transactions on Computers, 51(5), 541-552. doi:10.1109/TC.2002.1004593

Mishra, D. (2017). Efficient and Secure two-factor Dynamic ID-based Password Authentication Scheme with Provable Security. Cryptologia. Advance online publication. doi:10.1080/01611194.2017.1325787

Qiu, S., Xu, G., Ahmad, H., \& Guo, Y. (2018). An Enhanced Password Authentication Scheme for Session Initiation Protocol with Perfect Forward Secrecy. PLoS One, 13(3), e0194072. doi:10.1371/journal.pone.0194072 PMID:29547619

Shrivastava, G., Sharma, K., \& Bawankan, A. (2012). A New Framework Semantic Web Technology based e-learning. Environment and Electrical Engineering (EEEIC), $11^{\text {th }}$ International Conference on IEEE, 10171021. doi:10.1109/EEEIC.2012.6221527

Tang, Y. L., Hwang, M. S., \& Lee, C. C. (2002). A Simple Remote User Authentication Scheme. Mathematical and Computer Modelling, 36(1-2), 103-107. doi:10.1016/S0895-7177(02)00106-1

Tu, H., Kumar, N., Chilamkurti, N., \& Rho, S. (2015). An Improved Authentication Protocol for Session Initiation Protocol using Smart Card. Peer-to-Peer Net. Appl., 8(5), 903-910.

Wang, D., He, D., Wang, P., \& Chu, C. (2015). Anonymous two factor Authentications in Distributed Systems: Certain Goals are beyond Attainment. IEEE Transaction of Security \& Computationality, 12(4), 428-442. doi:10.1109/TDSC.2014.2355850

Wang, Y. Y., Liu, J. Y., Xiao, F. X., \& Dan, J. (2009). A More Efficient and Secure Dynamic ID-based Remote User Authentication Scheme. Computer Communications, 32(4), 583-585. doi:10.1016/j.comcom.2008.11.008

Xiong, L., Niu, J. W., Liu, Y., Liao, J., \& Liang, W. (2014). Robust Dynamic ID-based Remote User Authentication Scheme using Smart Cards. International Journal of Ad Hoc and Ubiquitous Computing, 17(4), 254-264. doi:10.1504/IJAHUC.2014.066423

Ajay Kumar Sahu is an Assistant Professor in the Department of Computer Science \& Engineering at Raj Kumar Goel Institute of Technology \& Management (RKGITM), Ghaziabad, affiliated to Dr. Abdul Kalam Technical University, Luck now (AKTU), Uttar Pradesh, India. His area of research interest includes Global Information Systems, Organizational Impact of IT, Software Development, Cloud Computing, Network Security and Software testing. He received his M. Tech degree from Guru Gobind Singh Indraprstha University (GGSIPU), Delhi in 2009 and B. Tech. (CSE) from G.L.A institute of Technology, Mathura in 2003. He has total Fifteen years of teaching experience in Academics. He has published more than ten research papers in international journals and proceedings.

Ashish Kumar specializes in Mobile AdHoc Network. He completed his B. Tech from Vikram University-Ujjain, and earned M. Tech from IIT-Kharagpur in Computer Science Engineering. He was awarded Ph. D. from UPES with accolades. His area of research focuses upon investigation of energy consumption in MANET. He has over one-and-a-half-decade experience in training in Object Oriented Techniques with UML, Operating Systems, Software Engineering, Mobile Computing and Distributed System. His interest is in providing sustainable solutions in the field of MANET, Reverse Engineering and Object Oriented Design. Dr. Ashish is a life member of IACSIT, IAENG, and ISTE. 